\title{
Internalizing and externalizing problems in obese children and adolescents: associations with daily salivary cortisol concentrations
}

\author{
Panagiota Pervanidou, ${ }^{1}$ Despoina Bastaki, ${ }^{1}$ Giorgos Chouliaras, ${ }^{1}$ \\ Katerina Papanikolaou, ${ }^{2}$ Christina Kanaka-Gantenbein, ${ }^{1}$ George Chrousos $^{1}$
}

${ }^{1}$ First Department of Pediatrics, 2Department of Child Psychiatry, University of Athens, School of Medicine, "Aghia Sophia" Children's Hospital, Athens, Greece

\begin{abstract}
OBJECTIVE: Pediatric obesity commonly co-exists with emotional and behavioral disorders, while disturbed cortisol concentrations have been reported in both obese and chronically stressed individuals with anxiety and/or depression. We investigated the prevalence of internalizing and externalizing problems, reported by both parents and children, in a clinical population of obese children (OC) compared to normal-weight children. We examined the role of cortisol as a potential mediator between obesity and such problems. DESIGN: We compared 110 obese with 31 normal-weight children. The Greek version of the child behavior checklist (CBCL) and the youth self-report (YSR) were used and salivary cortisol was determined serially five times a day. RESULTS: T-scores of internalizing problems (anxiety/depression, social withdrawal, somatic complains) reported by both children $(49.3 \pm 12.3 \mathrm{vs.} 43.2 \pm 9.1)$ and mothers $(60.6 \pm 11.3$ vs. 50.6 \pm 10.4$)$ were significantly higher $(p=0.03$ and $p<0.001$, respectively) in the obese than in the lean children. Externalizing problems (delinquency, rule-breaking behaviors) reported only by mothers were significantly higher in the OC $(57.2 \pm 10.5 \mathrm{vs} .48 .2 \pm 13.3, p=0.003)$. The cortisol area under the curve (AUC) was significantly smaller $(p=0.03)$ in the OC than in the controls; however, a cortisol correlation with internalizing/externalizing symptoms was not observed. CONCLUSIONS: There is a high prevalence of internalizing and externalizing problems in a clinical population of $\mathrm{OC}$. A mediation effect of cortisol in the relation between internalizing/ externalizing problems and obesity could not be supported.
\end{abstract}

Key words: Anxiety, Behavior, Children, Cortisol, Obesity, Stress

Address for correspondence:

Panagiota Pervanidou, Assistant Professor of Developmental and Behavioral Pediatrics, First Department of Pediatrics, University of Athens Medical School, Thivon \& Levadias Str., Athens, Greece, Tel.: +30 210 7467457, Fax: +30 2107759167 , E-mail: ppervanid@med.uoa.gr

Received: 23-11-2014, Accepted: 11-05-2015

\section{INTRODUCTION}

Childhood obesity has been recognized as one of the most serious health challenges of the $21^{\text {st }}$ century ${ }^{1}$ with a variety of health-related complications, such as metabolic syndrome manifestations (central adiposity, arterial hypertension, insulin resistance, 
dyslipidemia) and diabetes mellitus type $2 .{ }^{2,3}$ Recent evidence strongly indicates that both pediatric and adolescent obesity commonly co-exists with anxiety and depression, while a variety of behavioral problems have also been linked to increased weight status in children. ${ }^{4-6}$ Indeed, in a general population sample of 9-16 year-old children, studied longitudinally, chronic obesity was associated with psychopathology, more specifically, oppositional defiant disorder in both sexes and depressive disorder in males. ${ }^{4}$

Obesity results from an imbalance between energy intake and energy expenditure; however, hyperphagia is commonly a sign of anxiety, depression or impulsivity. In an epidemiologic sample, children and adolescents in the highest quartiles of body mass index (BMI) had a higher prevalence of depression, meaning that associations between these two conditions are more likely to co-exist in individuals with more severe obesity. ${ }^{7}$ In the opposite direction, anxiety disorders and depression in children followed up to adulthood were associated with a higher body weight in girls than controls without such symptomatology, while weights were higher in adolescents and young adults in whom depression was present at an early age. ${ }^{8}$ Furthermore, in a community sample of adolescents, both increased appetite and depressed mood were associated with recurrence of depression in adulthood. ${ }^{9}$

In addition, externalizing behaviors - such as hyperactivity, aggression or delinquency — have also been associated with increased weigh status in children as young as 24 months of age..$^{5,10,11}$ Other observational studies produced similar results: in a longitudinal study, a higher percentage of teacher-reported conduct problems was noted in overweight preschool children than in their normal-weight peers. ${ }^{12}$ However, an earlier study revealed no association between behavioral problems and obesity at the age of 5 years, while at age 14 overweight girls had over twice the odds of concurrent total behavioral problems, with no such association noted in boys. ${ }^{13}$ Impulsivity was also associated with overweight and weight-loss treatment failure among 9-year-old girls. ${ }^{14}$ One study reported that early childhood prevention efforts which targeted parenting practices for child behavior problems reduced these behaviors and lowered rates of obesity. ${ }^{15}$

Both emotional-behavioral and biological path- ways may mediate associations between childhood obesity and internalizing/externalizing problems. ${ }^{16}$ Lifestyle and behavioral parameters include poor adherence to self-care activities, sedentary habits and lack of physical exercise. ${ }^{17}$ Biologic mechanisms are mainly related to dysregulation of the stress system in which primarily the hypothalamic-pituitary-adrenal axis (HPA axis) and the sympathetic nervous system are involved. There is abundant evidence suggesting that children with symptoms of anxiety/depression are in most cases characterized by increased secretion of the stress hormones CRH and cortisol and the catecholamines norepinephrine and epinephrine. ${ }^{16}$ This hormonal state, when present on a chronic basis, could lead to central adiposity and the metabolic syndrome and also affect timing of puberty, physical growth and cognitive development. ${ }^{17,18}$

In this study we aimed to investigate the prevalence of internalizing (depression/anxiety, somatic complains and withdrawal) and externalizing (delinquent and aggressive) problems reported by both parents and children in a clinical population of obese children (OC) compared to normal-weight children. In addition, we examined the role of cortisol, as measured by five daily salivary samples, as a potential mediator between stress-related symptoms and obesity. We hypothesized a greater prevalence of internalizing and externalizing problems in obese than normal-weight children. Moreover, we hypothesized associations between these symptoms and daily salivary cortisol concentrations and a potential mediating role of cortisol.

\section{METHODS}

The study was approved by both the Scientific and the Ethics Committee of the "Aghia Sophia" Children's Hospital. Written informed consent was obtained from the participants and their parents. The study group consisted of children and adolescents evaluated at the Childhood Obesity Clinic of our Pediatrics Department. The comparison group consisted of normal-weight children recruited from the community by research advertisement. The recruitment was conducted according to the Helsinki Declaration. ${ }^{19}$ The study protocol was described and published previously, ${ }^{20}$ while a number of the children of the obese group (OG) had also participated in our previously published study. ${ }^{20}$ 


\section{Clinical evaluation and anthropometry}

BMI calculation: The children's BMI was calculated as their weight in kilograms divided by the square of their height in meters. Weight and height were measured during the physical examination. The status of obesity or overweight was defined based on Cole's international criteria. ${ }^{21}$ BMI standard deviations (SD) and z-scores were calculated based on the Greek growth charts for age and gender. ${ }^{22} \mathrm{~A}$ full clinical examination was performed by a certified pediatrician.

Pubertal assessment: Participants' pubertal development was determined via physical examination by a certified pediatrician based on the 5 Tanner Stages of pubic hair and genital development in boys and pubic hair and breast development in girls. ${ }^{23}$ Children with a Tanner Stage (for genital development in boys and breast development in girls) 2-4 were characterized as mid-pubertal and those with stage 5 as post-pubertal.

Exclusion criteria were: 1) underlying chronic illnesses, such as cardiac, hepatic and renal diseases; 2) chronic use of medications; 3 ) syndromic obesity; 4) mental disorders or pre-existing psychopathology; and 5) chromosomal disorders affecting puberty and/ or body weight.

\section{Psychometric instruments}

The Greek version of the child behavior checklist (CBCL) and the youth self-report (YSR) were used to assess internalizing and externalizing symptoms in children..$^{24,25}$ As conceived by Achenbach in 1991, ${ }^{26}$ internalizing symptoms refer to problems of withdrawal, somatic complaints and anxiety/depression, while externalizing symptoms manifest in delinquent and aggressive behaviors. Cronbach's alpha for internalizing and externalizing problems in the Greek version of the CBCL is 0.90 and 0.94 , respectively, whereas for both internalizing and externalizing problems in the Greek version of the YSR it is 0.90 . The data obtained through these two questionnaires were inserted into the ASEBA (Achenbach System of Empirically Based Assessment) computerized system ${ }^{27}$ and T-scores and percentiles of symptoms, based on Greek norms, were calculated. In addition, anxiety and depressive symptomatology were assessed using the Greek versions of the state-trait anxiety inventory for children (STAIC) and the children's depression inventory (CDI). The analysis of these two questionnaires is presented and discussed in our previous article with the same general cohort of children. ${ }^{20}$

Neuroendocrine evaluation: Salivary samples of the children were collected by their parents at home on the nearest Sunday to the physical and psychological examination day and were returned to the investigators within two days. The samples were collected five times a day (8.00 pre-breakfast, 12.00, 15.00, 18.00 and $21.00 \mathrm{hrs}$ pre-bed). The time of awakening was scheduled for 7.30 a.m. Detailed instructions were given to parents and children in order that they would collect the saliva samples correctly, using a Salivette device (Sarstedt, Nuembrecht, Germany). Participants were not receiving any medications. The children were told not to eat, drink, brush their teeth or exercise for at least half an hour prior to sample collection. Each of the samples was collected by having the participant place a cotton swab in his or her mouth for $2 \mathrm{~min}$ or by his/her chewing it for 1 $\mathrm{min}$. The cotton swab was then placed inside a plastic tube and kept in the refrigerator at $0-4{ }^{\circ} \mathrm{C}$. Within two days from collection, the samples were given to the investigator for further processing. Salivary cortisol was extracted from the cotton swab by centrifuging the plastic tubes and cotton at $1000 \mathrm{~g}$ for $8 \mathrm{~min}$ to separate off the saliva into the outer tube. The cotton swab was then removed and all samples were stored at $-85^{\circ} \mathrm{C}$. In addition, morning fasting blood samples were obtained for the measurement of serum cortisol. These samples were collected during a scheduled second visit to the Obesity Clinic, between 8 and 9 a.m. The blood samples were centrifuged and the serum obtained was stored at $-85^{\circ} \mathrm{C}$. Samples were processed using the Elecsys Cortisol reagent kit produced by ROCHE Co (Basel, Switzerland). Serum and salivary cortisol concentrations were measured using an electrochemiluminescence immunoassay (Roche Co., Basel, Switzerland). The intra-and interassay precision coefficients of variation for serum cortisol concentrations were 1.1-1.3 and $<8 \%$, while for salivary cortisol concentrations they ranged from 1.5-6.1 and 4.1-8\%, respectively. The analytical sensitivity (lower detection limit) for salivary cortisol concentrations was $<0.036 \mu \mathrm{g} / \mathrm{dl}$. 


\section{STATISTICAL ANALYSIS}

Continuous data are presented as mean $\pm \mathrm{SD}$, whereas categorical variables are presented as absolute (n) and relative (\%) frequencies. Comparisons of means were performed with the Student t-test. Relations between nominal variables were assessed by the Fisher exact test and correlations between continuous variables were estimated by Pearson's $r$. Non-parametric procedures (Kruskal-Wallis, MannWhitney and Spearman rho) were used whenever sample sizes were small $(<30)$. Linear regression analysis was used to adjust for the potential cofounding effect of age and gender on the relation between cortisol and obesity. All statistical procedures were run in the Stata 11.0 statistical software (StataCorp, 4905 Lakeway Drive College Station, Texas, 77845, USA).

\section{RESULTS}

Clinical characteristics (age, gender, BMI z-scores and pubertal status) of the study population are presented in Table 1. The children of the OG were younger than the comparison (normal-weight) Group (NWG).

T-scores of internalizing and externalizing symptoms were provided by the ASEBA based on the Greek norms. OC had a greater percentage of selfreported and mother-reported symptoms of internalizing symptoms categorized in the clinical range, as shown in Table 2. T-scores of internalizing symptoms expressed by the children themselves (using the YSR questionnaire) were significantly higher in the obese than the normal-weight children $(\mathrm{p}=0.03)$. The same was noted $(\mathrm{p}<0.001)$ for internalizing symptoms as assessed by mothers (using the CBCL). No significant differences were noted in externalizing symptoms as assessed by the children themselves. However, externalizing symptoms assessed by mothers were significantly higher in the obese than in the NWG $(p=0.003)$. These results are presented in Table 2 .

Cortisol area under the curve (AUC), as calculated by the five serial salivary samples for cortisol, was significantly higher $(\mathrm{p}=0.03)$ in the normal-weight than in the OG (Table 3). However, Pearson's correlation between cortisol AUC and age was significant $(r=0.3$, $\mathrm{p}=0.005)$. When gender was examined, differences in AUC between females $(0.625 \pm 0.485)$ and males $(0.536 \pm 0.292)$, as examined by the T-test, were not significant $(\mathrm{p}=0.3)$. In order to examine possible confounding effects of age and gender in the relation between cortisol and obesity, a linear regression analysis of cortisol AUC including age, gender and obesity was performed. The results, which are presented in Table 4, reveal that the significance of the relation between obesity and AUC was lost. In our data, this relation was probably due to the confounding effects of age and gender.

Lastly, associations between the children's AUC and internalizing-externalizing symptoms, expressed by both children and mothers, were not significant. Additional comparisons of AUC between children with high and low symptoms, expressed by both children and mothers, did not reveal significant results. Therefore, a mediation effect of cortisol in the relation of internalizing/externalizing disorders with obesity could not be supported by our data.

\section{DISCUSSION}

Our study revealed an increased prevalence of internalizing (withdrawal, somatic complaints and

Table 1. Characteristics of the Study Population

\begin{tabular}{lcccc}
\hline & & \multicolumn{2}{c}{ Obesity Status (according to Cole et al, 2000) } \\
\cline { 3 - 4 } & Total (n=141) & $\begin{array}{c}\text { Normal Weight Group } \\
\text { [NWG] (n=31) }\end{array}$ & $\begin{array}{c}\text { Obese Group } \\
\text { [OG] (n=110) }\end{array}$ & p-value \\
\hline Age (years, mean \pm SD) & $11.3 \pm 2.2$ & $13.0 \pm 1.5$ & $10.9 \pm 2.2$ & $<0.001$ \\
Gender, females, $\%$ & $51.8 \%$ & $58.6 \%$ & $50.0 \%$ & 0.5 \\
BMI z-score (mean \pm SD) & $2.47 \pm 1.81$ & $-0.13 \pm 0.60$ & $3.17 \pm 1.32$ & $<0.001$ \\
Pubertal status, pre-/mid-/postpubertal, $\mathrm{n}(\%)$ & $41.3 \% / 40.6 \% / 18.1 \%$ & $3.5 \% / 51.7 \% / 44.8 \%$ & $51.4 \% / 37.6 \% / 11.0 \%$ & $<0.001$ \\
\hline
\end{tabular}

***Fisher's exact test 
Table 2. Internalizing and Externalizing Problems, in T-scores and classification, reported by both child and mother, in obese and normal weight children (Obesity Status according to Cole et al)

\begin{tabular}{|c|c|c|c|c|}
\hline & \multirow[b]{2}{*}{ Total } & \multicolumn{2}{|c|}{ Obesity Status } & \multirow[b]{2}{*}{ p-value } \\
\hline & & Normal Weight Group & Obese Group & \\
\hline \multicolumn{5}{|l|}{ Internalizing, child } \\
\hline T-score & $48.3 \pm 11.9$ & $43.2 \pm 9.1$ & $49.3 \pm 12.3$ & 0.03 \\
\hline $\begin{array}{l}\text { classification: } \\
\text { normal, borderline, clinical (\%) }\end{array}$ & $80.3 \%, 8.2 \%, 11.5 \%$ & $96.2 \% / 3.8 \% / 0 \%$ & $76.0 \%, 9.4 \%, 14.6 \%$ & 0.048 \\
\hline \multicolumn{5}{|l|}{ Internalizing, mother } \\
\hline T-score & $58.8 \pm 11.4$ & $50.6 \pm 10.4$ & $60.6 \pm 11.3$ & $<0.001$ \\
\hline $\begin{array}{l}\text { classification: } \\
\text { normal, borderline, clinical (\%) }\end{array}$ & $54.2 \%, 11.7 \%, 34.1 \%$ & $83.3 \%, 4.2 \%, 12.5 \%$ & $46.9 \%, 13.5 \%, 39.6 \%$ & 0.006 \\
\hline \multicolumn{5}{|l|}{ Externalizing, child } \\
\hline T-score & $47.2 \pm 11.0$ & $45.0 \pm 10.4$ & $47.8 \pm 11.5$ & 0.28 \\
\hline $\begin{array}{l}\text { classification: } \\
\text { normal, borderline, clinical (\%) }\end{array}$ & $85.3 \%, 4.9 \%, 9.8 \%$ & $88.5 \%, 7.7 \%, 3.8 \%$ & $84.4 \% / 4.2 \% / 11.4 \%$ & 0.38 \\
\hline \multicolumn{5}{|l|}{ Externalizing, mother } \\
\hline T-score & $55.2 \pm 11.1$ & $48.2 \pm 13.3$ & $57.2 \pm 10.5$ & 0.003 \\
\hline $\begin{array}{l}\text { classification: } \\
\text { normal, borderline, clinical (\%) }\end{array}$ & $64.2 \%, 14.2 \%, 21.7 \%$ & $75.0 \%, 16.7 \%, 8.3 \%$ & $61.5 \%, 13.5 \%, 25.0 \%$ & 0.18 \\
\hline
\end{tabular}

Table 3. Cortisol distribution in the study population according to Obesity Status (Cole et al, 2000)

\begin{tabular}{lcccc}
\hline & & \multicolumn{2}{c}{ Obesity Status } & \\
\cline { 2 - 4 } & Total & Normal Weight Group & Obese Group & p-value \\
\hline Cortisol AUC & $0.581 \pm 0.402$ & $0.696 \pm 0.251$ & $0.558 \pm 0.424$ & 0.03 \\
\hline
\end{tabular}

Table 4. Linear regression of Cortisol AUC, including age, gender and Obesity Status (Cole et al., 2000) as covariates

\begin{tabular}{lcc}
\hline & $\boldsymbol{\beta}$-coefficient (95\% ci) & p-value \\
\hline Age & $0.062(0.021,0.103)$ & 0.003 \\
Gender (males vs. females) & $-0.161(-0.335,0.011)$ & 0.067 \\
$\begin{array}{l}\text { Obesity Status } \\
\text { (obese vs. Normal weight) }\end{array}$ & $-0.004(-0.241,0.233)$ & 0.972 \\
\hline
\end{tabular}

anxiety/depression) and externalizing (delinquent and aggressive behaviors) problems in a clinical population of $\mathrm{OC}$, in comparison to normal weight children. Increased T-scores of internalizing problems in our sample were expressed by both mothers and children, whereas increased T-scores of externalizing problems were reported only by mothers. Daily salivary cortisol concentrations were lower in the obese than in the control group; however, the significance was lost when age and gender were inserted in the analysis. No associations were found between cortisol and internalizing-externalizing problems.

Our study is in accordance with epidemiological studies showing an increased prevalence of mental health problems in OC. $4,5,7,28$ The OC in our study are derived from a Childhood Obesity Clinic, supporting previous evidence that treatment seekers (clinical samples) are more likely to have disturbed eating behaviors than overweight youngsters from community samples. ${ }^{29}$ Several mechanisms link internalizing problems and obesity in children: poor adherence to self-care activities and sedentary habits such as excessive television viewing and internet use. ${ }^{17}$ Disturbed eating behaviors, such as emotional and external eating patterns, are potential mediators of this association. ${ }^{30}$ In addition, low self-esteem and feelings of guilt and personal failure characterize both obesity and internalizing problems..$^{31,32}$ Furthermore, the obesogenic effect of reduced sleep is thought to 
be mediated by both behavioral and hormonal factors. Children have more time to eat, and sleep deprivation reduces leptin and raises ghrelin levels, which increase appetite..$^{33}$ Externalizing problems in children are by definition assessed by parents' and teachers' reports and not by the reports of children themselves, which is consistent with our findings. Attention deficit hyperactivity disorder, the most prevalent behavioral disorder in childhood, is highly comorbid with other externalizing problems and has been linked to obesity in children. ${ }^{34-37}$ Underlying mechanisms linking externalizing problems with pediatric obesity include: impulsive behaviors related to uncontrolled eating, ${ }^{37}$ elevated sensitivity to immediate reward ${ }^{38}$ and impaired executive functions (neurocognitive skills that are necessary for planning, monitoring and executing goal-directed actions) that might foster dysregulated eating behaviors, such as binge eating, emotional eating or eating in the absence of hunger. ${ }^{39}$ Children with externalizing behaviors may also be at greater risk of food addictions. Lastly, externalizing problems and obesity may share common genetic and neurobiologic dysfunctions, involving the dopaminergic and possibly other systems. ${ }^{40}$

The HPA axis, in addition to other biologic mechanisms, may mediate both internalizing and externalizing behaviors in childhood obesity, since anxiety disorders and depression have been linked to HPA axis dysregulation in adults and children. ${ }^{41-44}$ However, relatively few studies have examined associations between anxiety/depression, the HPA axis and obesity. ${ }^{20,45-47}$ Our previous study, carried out in a clinical population of $\mathrm{OC},{ }^{20}$ revealed that overall salivary cortisol concentrations were increased in children with anxiety or depressive symptomatology, as assessed by the STAIC and the CDI questionnaires ${ }^{48,49}$ compared to OC without any affective morbidity and to those with anxiety and depression comorbidity. Disturbed cortisol concentrations in these children might be associated with further morbidity. In the present study, we did not find any differences in daily cortisol concentrations between children with and without internalizing problems. This finding may be due to the fact that "internalizing problems" of CBCL involve a wider range of symptoms compared to those of STAIC and CDI. The HPA axis has also recently been investigated in children with externalizing prob- lems. ${ }^{50-55}$ There is a body of evidence demonstrating an association between altered HPA axis reactivity and aggressive behavior. Several studies have shown reduced cortisol concentrations and a blunted HPA axis reactivity to stress. ${ }^{56-59}$ No evidence has so far linked childhood obesity, externalizing symptoms and cortisol concentrations. In our study, we investigated but did not establish such associations, possibly due to the fact that only problem-scales, and not clinical psychiatric disorders, were investigated. In addition, basal cortisol concentrations, relative to stress-induced cortisol concentrations, are a weaker measure of HPA activity in children. ${ }^{60}$

Finally, we found that $\mathrm{OC}$, in general, had a lower cortisol AUC compared to normal-weight children. This finding is consistent with a recent school-based study showing lower daily basal cortisol concentrations in obese than in normal-weight children. ${ }^{61}$ In this study, cortisol levels did not vary significantly with age or sex. A small number of other studies ${ }^{62-64}$ examining either basal cortisol levels or cortisol responses to stress confirm these results; however, other studies do not. ${ }^{65,66}$ In our sample, although OC had a significantly lower AUC compared to controls, this difference was lost after inserting the effect of age. A larger sample size of normal weight children could provide better evidence.

The main strength of the study is the fact that, in contrast to school-based or epidemiological studies, all children were examined in a clinical setting by pediatricians specialized in pediatric obesity. Limitations of our study are the cross-sectional design and the mild age difference in our groups. However, T-scores (for age and gender), and not absolute numbers, of internalizing/externalizing problems were used; thus, age and gender were incorporated in T-score values allowing comparisons between the two groups. Furthermore, the possible confounding effect of age and gender on the relation between obesity and cortisol was taken into account by inserting age and gender as co-variates in the multivariate regression analysis. Thus, differences in age and gender between the two groups, the obese and the normal weight, did not affect the interpretation of the results. Not all scales of the CBCL and the YSR were analyzed. In order to avoid repetitions and overlaps between symptom-scales, we chose to 
analyze only the two wide scales of internalizing and externalizing problems. Lastly, no behavioral-emotional mechanisms, such as emotional and/or external eating, were taken into account. Future research carried out in clinical populations of OC are likely to overcome these limitations thus providing more insight into the biologic and behavioral mechanisms linking obesity to psychosocial and mental health.

\section{DISCLOSURE STATEMENT}

The authors have nothing to disclose. There is no conflict of interest.

\section{ACKNOWLEDGMENTS}

We thank the families who participated in the study.

\section{REFERENCES}

1. World Health Organization (2012) Global strategy on diet, physical activity and health. 2012. Available at: http://www.who.

2. Weiss R, Dziura J, Burgert TS, 2004 Obesity and Metabolic Syndrome in Children and Adolescents. N Engl J Med 350: 2362-2374.

3. Kassi E, Pervanidou P, Kaltsas G, Chrousos G, 2011 Metabolic syndrome: definitions and controversies. BMC Med 9: 48.

4. Mustillo S, Worthman C, Erkanli A, Keeler G, Angold A, Costello EJ, 2003 Obesity and psychiatric disorder: developmental trajectories. Pediatrics 111: 851-859.

5. Anderson S, He X, Schoppe-Sullivan S, et al, 2010 Externalizing behavior in early childhood and body mass index from age 2 to 12 years: longitudinal analyses of a prospective cohort study. BMC Pediatr 10: 49.

6. Sanderson K, Patton GC, McKercher C, Dwyer T, Venn AJ, 2011 Overweight and obesity in childhood and risk of mental disorder: a 20-year cohort study. Aust N Z J Psychiatry 45: 84-92.

7. Onyike CU, Crum RM, Lee HB, Lyketsos CG, Eaton WW, 2003 Is obesity associated with major depression? Results from the Third National Health and Nutrition Examination Survey. Am J Epidemiol 158: 1139-1147.

8. Anderson SE, Cohen P, Naumova EN, Must A, 2006 Association of depression and anxiety disorders with weight change in a prospective community-based study of children followed up into adulthood. Arch Pediatr Adolesc Med 160: 285-291.

9. Pettit JW, Lewinsohn PM, Joiner TE Jr, 2006 Propagation of major depressive disorder: relationship between first episode symptoms and recurrence. Psychiatry Res 141: 271-227.
10. Puder JJ, Munsch S, 2010 Psychological correlates of childhood obesity. Int J Obes (Lond) 34: Suppl 2: 37-43.

11. Pauli-Pott U, Neidhard J, Heinzel-Gutenbrunner M, Becker K, 2014 On the link between attention deficit/ hyperactivity disorder and obesity: do comorbid oppositional defiant and conduct disorder matter? Eur Child Adolesc Psychiatry 23: 531-537.

12. Sawyer MG, Miller-Lewis L, Guy S, Wake M, Canterford L, Carlin JB, 2006 Is there a relationship between overweight and obesity and mental health problems in 4- to 5-year-old Australian children? Ambul Pediatr 6: 306-311.

13. Lawlor DA, Mamun AA, O'Callaghan MJ, Bor W, Williams GM, Najman JM, 2005 Is being overweight associated with behavioural problems in childhood and adolescence? Findings from the Mater-University study of pregnancy and its outcomes. Arch Dis Child 90: 692-697.

14. Nederkoorn C, Jansen E, Mulkens S, et al, 2007 Impulsivity predicts treatment outcome in obese children. Behav Res Ther 45: 1071-1075.

15. Brotman LM, Dawson-McClure S, Huang KY, et al, 2012 Early childhood family intervention and long-term obesity prevention among high- risk minority youth. Pediatrics 129: e621-e628.

16. Pervanidou P, Chrousos GP, 2011 Stress and obesity/ metabolic syndrome in childhood and adolescence. Int J Pediatr Obes 6: Suppl 1: 21-28.

17. Pervanidou P, Chrousos GP, 2012 Metabolic consequences of stress during childhood and adolescence. Metabolism 61: 611-619.

18. Chrousos GP, 2009 Stress and disorders of the stress system. Nat Rev Endocrinol 5: 374-381.

19. Williams JR, 2008 The Declaration of Helsinki and public health. Bulletin of the World Health Organization 86: 650-651.

20. Pervanidou P, Bastaki D, Chouliaras G, et al, 2013 Circadian cortisol profiles, anxiety and depressive symptomatology, and body mass index in a clinical population of obese children. Stress 16: 34-43.

21. Cole TJ, Bellizzi MC, Flegal KM, Dietz WH, 2000 Establishing a standard definition for child overweight and obesity worldwide: international survey. BMJ 320 : 1240-1243.

22. Chiotis D, Krikos X, Tsiftis G, Hatzisymeaon M, ManiatiChristidi M, Dacou-Voutetakis C, 2004 Body mass index and prevalence of obesity in subjects of Hellenic origin aged 0-18 years, living in the Athens area. Ann Clin Paediatr Univ Atheniensis 51: 139-154.

23. Tanner JM, 1962 Growth at adolescence. Blackwell Scientific Publications, Oxford.

24. Roussos A, Karantanos G, Richardson C, et al, 1999 Achenbach's Child Behavior Checklist and Teachers' Report Form in a normative sample of Greek children 6-12 years old. Eur Child Adolesc Psychiatry 8: 165-172.

25. Roussos A, Francis K, Zoubou V, Kiprianos S, Pro- 
kopiou A, Richardson C, 2001 The standardization of Achenbach's Youth Self-Report in Greece in a national sample of high school students. Eur Child Adolesc Psychiatry 10: 47-53.

26. Achenbach TM, 1991 Manual for Child Behavior Checklist/4-18 and 1991 Profile. Burlington, VT: University of Vermont.

27. Achenbach TM, 2009 The Achenbach System of Empirically Based Assessment (ASEBA): Development, Findings, Theory, and Applications. Burlington, VT. University of Vermont Research Center for Children, Youth and Families.

28. Goldbacher EM, Matthews KA, 2007 Are Psychological Characteristics Related to Risk of the Metabolic Syndrome? A Review of the Literature. Annals of Behavioral Medicine 34: 240-252.

29. Goossens L, Braet C, Van Vlierberghe L, et al, 2009 Loss of control over eating in overweight youngsters: the role of anxiety, depression and emotional eating. Eur Eat Disord Rev 17: 68-78.

30. Harrist AW, Hubbs-Tait L, Topham GL, Shriver LH, Page MC, 2013 Emotion regulation is related to children's emotional and external eating. J Dev Behav Pediatr 34: 557-565.

31. Sjöberg RL, Nilsson KW, Leppert J, 2005 Obesity, shame, and depression in school-aged children: a populationbased study. Pediatrics 116: e389-392.

32. Mond J, van den Berg P, Boutelle K, Hannan P, Neumark-Sztainer D, 2011 Obesity, body dissatisfaction, and emotional well-being in early and late adolescence: findings from the project EAT study. J Adolesc Health 48: 373-378.

33. Hart CN, Carskadon MA, Considine RV, et al, 2013 Changes in children's sleep duration on food intake, weight, and leptin. Pediatrics 132: e1473-1480.

34. Fliers EA, Buitelaar JK, Maras A, et al, 2013 ADHD is a risk factor for overweight and obesity in children. J Dev Behav Pediatr 34: 566-574.

35. Erhart M, Herpertz-Dahlmann B, Wille N, SawitzkyRose B, Hölling H, Ravens-Sieberer U, 2012 Examining the relationship between attention-deficit/hyperactivity disorder and overweight in children and adolescents. Eur Child Adolesc Psychiatry 21: 39-49.

36. Cortese S, Morcillo Peñalver C, 2010 Comorbidity between ADHD and obesity: exploring shared mechanisms and clinical implications. Postgrad Med 122: 88-96.

37. Graziano PA, Bagner DM, Waxmonsky JG, Reid A, McNamara JP, Geffken GR, 2012 Co-occurring weight problems among children with attention deficit/hyperactivity disorder: the role of executive functioning. Int J Obes (Lond) 36: 567-572.

38. Luman M, van Meel CS, Oosterlaan J, et al, 2012 Reward and punishment sensitivity in children with ADHD: validating the sensitivity to punishment and sensitivity to reward questionnaire for children (SPSRQ-C). J Abnorm Child Psychol 40: 145-157.
39. Cortese S, Comencini E, Vincenzi B, Speranza M, Angriman M, 2013 Attention-deficit/hyperactivity disorder and impairment in executive functions: a barrier to weight loss in individuals with obesity? BMC Psychiatry 13: 286.

40. Cortese S, Vincenzi B, 2012 Obesity and ADHD: Clinical and Neurobiological Implications. Curr Top Behav Neurosci 9: 199-218.

41. Pervanidou P, 2008 Biology of Posttraumatic Stress Disorder in childhood and adolescence J Neuroendocrinol 20: 632-638.

42. Pervanidou P, Chrousos GP, 2010 Neuroendocrinology of Posttraumatic Stress Disorder. Prog Brain Res 182: 149-160.

43. Nelemans SA, Hale WW 3rd, Branje SJ, et al, 2014 Persistent Heightened Cortisol Awakening Response and Adolescent Internalizing Symptoms: A 3-year Longitudinal Community Study. J Abnorm Child Psychol 42: 767-777

44. Ulrike S, Reinhold L, Dirk H, 2013 Major depression in young girls is related to altered cortisol awakening response. Eur Child Adolesc Psychiatry 22: 379-384.

45. Hillman JB, Dorn LD, Bin Huang, 2010 Association of anxiety and depressive symptoms and adiposity among adolescent females, using dual energy X-ray absorptiometry. Clin Pediatr (Phila) 49: 671-677.

46. Hillman JB, Dorn LD, Loucks TL, Berga SL, 2012 Obesity and the hypothalamic-pituitary-adrenal axis in adolescent girls. Metabolism 61: 341-348.

47. Dockray S, Susman EJ, Dorn LD, 2009. Depression, cortisol reactivity, and obesity in childhood and adolescence. J Adolesc Health 45: 344-350.

48. Psychountaki M, Zervas Y,Karteroliotis K, Spielberger C, 2003 Reliability and Validity of the Greek Version of the STAIC. Eur J Psychol Assess 19: 124-130.

49. Giannakopoulos G, Kazantzi M, Dimitrakaki C, Tsiantis J, Kolaitis G, Tountas Y, 2009 Screening for children's depression symptoms in Greece: the use of the Children's Depression Inventory in a nation-wide school-based sample. Eur Child Adolesc Psychiatry 18: 485-492.

50. Isaksson J, Nilsson KW, Lindblad F, 2013 Early psychosocial adversity and cortisol levels in children with attention-deficit/hyperactivity disorder. Eur Child Adolesc Psychiatry 22: 425-432.

51. von Polier GG, Herpertz-Dahlmann B, Konrad K, et al, 2013 Reduced cortisol in boys with early-onset conduct disorder and callous-unemotional traits. Biomed Res Int 2013: 349530.

52. Isaksson J, Nilsson KW, Nyberg F, Hogmark A, Lindblad F, 2012 Cortisol levels in children with attention-deficit/ hyperactivity disorder. J Psychiatr Res 46: 1398-1405.

53. Yang SJ, Shin DW, Noh KS, Stein MA, 2007 Cortisol is inversely correlated with aggression for those boys with attention deficit hyperactivity disorder who retain their reactivity to stress. Psychiatry Res 153: 55-60.

54. Fairchild G, 2012 Hypothalamic-pituitary-adrenocortical 
axis function in attention-deficit hyperactivity disorder. Curr Top Behav Neurosci 9: 93-111.

55. Feilhauer J, Cima M, Korebrits A, Nicolson NA, 2013 Salivary cortisol and psychopathy dimensions in detained antisocial adolescents. Psychoneuroendocrinology 38: 1586-1595.

56. Stadler C, Kroeger A, Weyers P, et al, 2011 Cortisol reactivity in boys with attention-deficit/hyperactivity disorder and disruptive behavior problems: the impact of callous unemotional traits. Psychiatry Res 187: 204-209.

57. Platje E, Vermeiren RR, Raine A, et al, 2013 A longitudinal biosocial study of cortisol and peer influence on the development of adolescent antisocial behavior. Psychoneuroendocrinology 38: 2770-2779.

58. Platje E, Jansen LM, Raine A, et al, 2013 Longitudinal associations in adolescence between cortisol and persistent aggressive or rule-breaking behavior. Biol Psychol 93: 132-137.

59. Haltigan JD, Roisman GI, Susman EJ, Barnett-Walker K, Monahan KC, 2011 Elevated trajectories of externalizing problems are associated with lower awakening cortisol levels in midadolescence. Dev Psychol 47: 472-478.

60. Fairchild G, van Goozen SH, Stollery SJ, et al, 2008 Cortisol diurnal rhythm and stress reactivity in male adolescents with early-onset or adolescence-onset conduct disorder. Biol Psychiatry 64: 599-606.

61. Kjölhede EA, Gustafsson P, Gustafsson P, Nelson N, 2014 Overweight and obese children have lower cortisol levels than normal weight children. Acta Paediatr 103: 295-299.

62. Lumeng JC, Miller A, Peterson KE, et al, 2014 Diurnal cortisol pattern, eating behaviors and overweight in lowincome preschool-aged children. Appetite 73: 65-72.

63. Miller AL, Clifford C, Sturza J, et al, 2013 Blunted cortisol response to stress is associated with higher body mass index in low-income preschool-aged children. Psychoneuroendocrinology 38: 2611-2617.

64. Ruttle PL, Javaras KN, Klein MH, et al, 2013 Concurrent and longitudinal associations between diurnal cortisol and body mass index across adolescence. J Adolesc Health 52: 731-737.

65. Abraham SB, Rubino D, Sinaii N, Ramsey S, Nieman LK, 2013 Cortisol, obesity, and the metabolic syndrome: a cross-sectional study of obese subjects and review of the literature. Obesity (Silver Spring) 21: E105-117.

66. Veldhorst MA, Noppe G, Jongejan MH, et al,2014 Increased scalp hair cortisol concentrations in obese children. J Clin Endocrinol Metab 99: 285-290. 